\title{
Identification of single nucleotide polymorphism markers associated with bacterial cold water disease resistance and spleen size in rainbow trout
}

OPEN ACCESS

Edited by:

Peng $X u$,

Chinese Academy of Fishery

Sciences, China

Reviewed by:

Ross Houston,

University of Edinburgh, UK

Lior David,

The Hebrew University of Jerusalem,

Israel

*Correspondence: Sixin Liu,

National Center for Cool and Cold

Water Aquaculture, Agricultural

Research Service, United States

Department of Agriculture,

11861 Leetown Road, Kearneysville,

WV 25430, USA

sixin.liu@ars.usda.gov

tPresent address:

David P. Marancik,

Fish Vet Group, Portland, ME, USA

Specialty section:

This article was submitted to

Livestock Genomics,

a section of the journal

Frontiers in Genetics

Received: 16 July 2015 Accepted: 09 September 2015 Published: 24 September 2015

Citation:

Liu S, Vallejo RL, Palti Y, Gao G, Marancik DP, Hernandez AG and Wiens GD (2015) Identification of single nucleotide polymorphism markers associated with bacterial cold water disease resistance and spleen size in rainbow trout.

Front. Genet. 6:298.

doi: 10.3389/fgene.2015.00298

\section{Sixin Liu' ${ }^{*}$, Roger L. Vallejo', Yniv Palti', Guangtu Gao', David P. Marancik ${ }^{1+}$, Alvaro G. Hernandez ${ }^{2}$ and Gregory D. Wiens ${ }^{1}$}

\author{
${ }^{1}$ National Center for Cool and Cold Water Aquaculture, Agricultural Research Service, United States Department of \\ Agriculture, Kearneysville, WW, USA, ${ }^{2}$ Roy J. Carver Biotechnology Center, University of Illinois at Urbana-Champaign, \\ Urbana, IL, USA
}

Bacterial cold water disease (BCWD) is one of the frequent causes of elevated mortality in salmonid aquaculture. Previously, we identified and validated microsatellites on chromosome Omy19 associated with QTL (quantitative trait loci) for BCWD resistance and spleen size in rainbow trout. Recently, SNPs (single nucleotide polymorphism) have become the markers of choice for genetic analyses in rainbow trout as they are highly abundant, cost-effective and are amenable for high throughput genotyping. The objective of this study was to identify SNP markers associated with BCWD resistance and spleen size using both genome-wide association studies (GWAS) and linkagebased QTL mapping approaches. A total of 298 offspring from the two half-sib families used in our previous study to validate the significant BCWD QTL on chromosome Omy19 were genotyped with RAD-seq (restriction-site-associated DNA sequencing), and 7,849 informative SNPs were identified. Based on GWAS, 18 SNPs associated with BCWD resistance and 20 SNPs associated with spleen size were identified. Linkagebased QTL mapping revealed three significant QTL for BCWD resistance. In addition to the previously validated dam-derived QTL on chromosome Omy19, two significant BCWD QTL derived from the sires were identified on chromosomes Omy8 and Omy25, respectively. A sire-derived significant QTL for spleen size on chromosome Omy2 was detected. The SNP markers reported in this study will facilitate fine mapping to identify positional candidate genes for BCWD resistance in rainbow trout.

Keywords: rainbow trout, Flavobacterium psychrophilum, bacterial cold water disease, spleen size, SNP, QTL

\section{Introduction}

Bacterial cold water disease (BCWD) is one of the frequent causes of elevated mortality in salmonid aquaculture (Nematollahi et al., 2003; Starliper, 2011; Loch and Faisal, 2015). The etiological agent of BCWD is a Gram-negative bacterium, Flavobacterium psychrophilum, which also causes rainbow trout fry syndrome in small fish. Currently, there is no licensed commercial vaccine for BCWD. Use of the limited antibiotics available in food fish can increase the production costs and is a concern 
for emergence of antibiotic-resistant pathogens. Fortunately, host resistance can be improved through selective breeding (Leeds et al., 2010), and a fall-spawning line with improved BCWD resistance evaluated in both laboratory and on-farm tests has been developed (Wiens et al., 2013a). Currently, there is interest in improving BCWD resistance of other populations of different origin and spawn times. Conventional family based selection for BCWD resistance relies on indirect phenotype evaluation of siblings, which does not take advantage of the genetic variation within families. The development of molecular markers associated with BCWD resistance would facilitate marker-assisted selection for BCWD resistance and enable direct selection of breeding candidates.

As the first step to implement marker assistant selection and eventual positional cloning of genes for BCWD resistance, we have conducted several studies to map QTL (quantitative trait loci) for BCWD resistance in rainbow trout. Because rainbow trout mature after 2 years of growth, there are two yearclass breeding populations: even-year population and odd-year population. Previously, we used microsatellites to identify QTL for BCWD resistance in either even or odd-year populations of rainbow trout. Nine major QTL on seven chromosomes were identified in six odd-year mapping families (Vallejo et al., 2014a). In an even year mapping family, 2008132, a major QTL for BCWD resistance on chromosome Omy19 was identified (Wiens et al., 2013b). Interestingly, a major QTL for spleen index on chromosome Omy19 was also identified in the same mapping family, which was consistent with the association between BCWD resistance and spleen size in rainbow trout reported in our previous study (Hadidi et al., 2008). Both QTL for BCWD resistance and spleen size on chromosome Omy19 were validated in a subsequent generation (Vallejo et al., 2014b), and the QTL mapping results suggest that two separate QTL on chromosome Omy19 control BCWD resistance and spleen size, respectively.

The microsatellites available in rainbow trout are limited, and microsatellite genotyping for whole-genome scans is no longer cost effective while the cost of SNP (single nucleotide polymorphism) genotyping is decreasing. SNPs have become the markers of choice as they are highly abundant and are amenable for high throughput genotyping. RAD-seq (restrictionsite-associated DNA sequencing) targets sequences surrounding the restriction enzyme cut site (Miller et al., 2007; Baird et al., 2008), which has rapidly become a cost-effective method of SNP genotyping in aquaculture species as it does not require a prior marker discovery or a reference genome sequence. Recently, Campbell et al. (2014) reported SNP markers associated BCWD resistance in rainbow trout using RAD-seq. Similarly, we have also used RAD-seq to identify SNPs associated with QTL for stress response (Liu et al., 2015) and for BCWD resistance in odd-year mapping families (Palti et al., 2015b). In this study, offspring of two dam half-sib even-year mapping families used previously for microsatellites-based validation of the QTL for BCWD resistance on chromosome Omy19 (Vallejo et al., 2014b) were genotyped using RAD-seq. The objective of this study was to identify SNPs associated with BCWD resistance and spleen size using both genome-wide association studies (GWAS) and linkage-based QTL mapping approaches.

\section{Materials and Methods}

\section{Ethic Statement}

The experiment was conducted in accordance of protocol \#47, approved by the Institutional Animal Care and Use Committee, National Center for Cool and Cold Water Aquaculture, Agricultural Research Service, United States Department of Agriculture.

\section{Mapping Families and Phenotyping}

Two dam half-sib mapping families 2012473 and 2012474 were used in this study. Family 2012473 was made from the cross between 2008132002 and 2009044043, and family 2012474 was made from the cross between 2008132002 and 2009044044. The 4-year-old female fish 2008132002 was derived from the mapping family 2008132 used previously for BCWD QTL mapping in even-year class fish (Wiens et al., 2013b). Both 3-year-old male fish 2009044043 and 2009044044 were derived from the mapping family 2009044 used previously for BCWD QTL mapping in oddyear class fish (Vallejo et al., 2014a). These two mapping families were chosen for this study because the shared dam 2008132002 is segregating for the BCWD QTL but not for the spleen index QTL on chromosome Omy19 (Vallejo et al., 2014b). In addition, the sire of family 2012474 (fish 2009044044) is segregating for the QTL for spleen index on chromosome Omy19 (Vallejo et al., 2014b).

The disease challenge and collection of phenotype data were described in our previous publication (Vallejo et al., 2014b). Briefly, 100 fish from each mapping family were challenged with BCWD strain CSF259-93 by intraperitoneal injection at an average of 62 days post-hatch. Mortalities were collected each day for 21 days after intraperitoneal injection. Survival days (DAYS), the number of days to death post challenge, were recorded and survivors at the end of the experiment were assigned a value of 22 days. Each individual fish also had a record of survival status (STATUS). The binary trait STATUS had two classes: fish died during the 21 days period post challenge were assigned a value of 1 , and fish alive on day 22 post-challenge were assigned a value of 2. Phenotypes DAYS and STATUS were used for GWAS and QTL mapping for BCWD resistance. Both body weight and spleen weight were recorded for 98 naïv 182-day-old fish from the family 2012474. Spleen index for each fish was calculated using the formula $2.1 \times$ [spleen weight $(\mathrm{mg}) /$ body weight $(\mathrm{g})$ ] (Vallejo et al., 2014b).

\section{RAD Genotyping}

Genomic DNA of three mapping parents and 298 offspring of mapping families 2012473 and 2012474 was digested with restriction enzyme SbfI, and $\mathrm{RAD}$ sequencing libraries were prepared as described in Palti et al. (2014). Each RAD library containing 30 to 32 indexed samples with a unique six-nucleotide barcode was sequenced (single end $100 \mathrm{bp}$ read) on a single lane of HiSeq 2000. Raw sequences were submitted to the short read archive of GenBank under accession number SRP062268. After trimming the six-base barcode at the $5^{\prime}$ end and the last five bases at the $3^{\prime}$ end of each sequence read, we filtered out reads with a cumulative sequencing error probability of more than $20 \%$ in the 
89 bp read (i.e., call probability better than $99.5 \%$ per base for the sequences that passed filtering). The remaining trimmed reads were processed to identify SNPs using Novoalign and Perl scripts (Palti et al., 2014). SNP loci and samples with more than 30\% missing data were removed from the final genotype data (passing call rate $>70 \%)$. Also, Chi-square goodness-of-fit tests were used to check the genotype segregation ratio (1:1 or $1: 2: 1)$ of each SNP, and SNPs with Bonferroni-corrected segregation distortions $(P<1 \mathrm{e}-5)$ were dropped from the final genotype dataset.

\section{Construction of SNP Linkage Maps}

The SNP genotypes of these two mapping families 2012473 and 2012474 were combined to construct linkage maps using software MULTIMAP (Matise et al., 1994). Based on two-point analysis, SNPs were assigned to 29 linkage groups with parameters of LOD 10 and recombination fractions less than 0.2 . The SNP sequences were mapped to the reference genome sequence of rainbow trout (Berthelot et al., 2014). Based on the chromosomal assignments of majority SNPs in each linkage group, the 29 linkage groups were assigned to chromosomes of rainbow trout. We used sire-specific and dam-specific SNPs in each linkage group to construct male and female genetic maps, respectively. Due to the large number of SNPs in each linkage groups, it was challenging to build linkage maps using directly the default threshold of LOD 3. Thus, the initial linkage map was constructed with a threshold of LOD 10. For each linkage group where the linkage map was smaller than $150 \mathrm{cM}$, more SNPs were added to the linkage map using a threshold of LOD 5. If the linkage group map length was still smaller than $150 \mathrm{cM}$ with the threshold of LOD 5, the threshold was lowered to LOD 3 to add more SNPs to the map.

\section{Genome Wide Association Analysis}

Single nucleotide polymorphism markers that were assigned to chromosomes through two-point linkage analysis and had over $70 \%$ genotype call rates were used for GWAS using the GrammarGamma method (Svishcheva et al., 2012) implemented in the R package GenABEL (Aulchenko et al., 2007). This method runs a single-point association test accounting for family relatedness and includes two analysis steps. First, we performed polygenic analysis modeling family relationships; the genomic kinship matrix estimated from SNPs was included in the model of association analysis. Second, we performed association analysis using the residuals from step 1 analysis. Thus, corrected SNPs effect were used in the association tests. We used the threshold $P<0.001$ to identify SNPs associated with the traits of interest. For simplicity, only SNPs significant for both survival days and survival status were defined as significant SNPs associated with BCWD resistance.

\section{QTL Mapping}

The half-sib regression analysis module implemented in software GridQTL (Seaton et al., 2006) was used to identify QTL for BCWD resistance and spleen index as described in our previous publication. Based on 10,000 permutations, a significant QTL has to be significant $(P<0.05)$ at both the chromosome-wide level and the experiment-wide level; a suggestive QTL is only significant $(P<0.05)$ at the chromosome-wide level but not at the experiment-wide level. The 95\% QTL confidence intervals were estimated using 10,000 bootstraps with re-sampling with software GridQTL (Seaton et al., 2006). The proportion of phenotypic variance explained by the QTL was calculated as $h_{q}{ }^{2}=4\left(1-M S E_{\text {full }} / M S E_{\text {reduced }}\right)$ where $\mathrm{MSE}_{\text {full }}$ and $\mathrm{MSE}_{\text {reduced }}$ are the mean squared error of the full and reduced model, respectively.

\section{Results}

\section{RAD Genotyping and SNP Linkage Maps}

Between 6.52 and 10.01 million quality filtered reads were obtained from each mapping parent, and a total of 7,849 SNPs (Supplementary File) were identified. The number of quality filtered reads per offspring ranged from 0.16 to 5.7 million with an average of 2.34 million. Among the 298 offspring genotyped with RAD-seq, only two fish used to measure spleen index had genotype call rates less than $70 \%$. Thus, these two fish were removed from subsequent data analysis. Based on twopoint linkage analysis, 7,595 SNPs were assigned to the 29 chromosomes of rainbow trout. The rest of SNPs were either unlinked with other SNPs or were assigned to small linkage groups with unknown chromosome assignments. A total of 638 SNPs were placed on the female linkage maps with a total map length of $5483 \mathrm{cM}$ and an average SNP spacing of $8.6 \mathrm{cM}$. Likewise, a total of 438 SNPs were placed on the male linkage maps with a total map length of $2964 \mathrm{cM}$ and an average SNP spacing of $6.8 \mathrm{cM}$. Due to the limitation of genetic mapping software, we used only dam-specific or sire-specific SNPs to construct female or male linkage maps, respectively. Also, to ensure high confidence in the location and order of the markers on each chromosome, very stringent LOD thresholds were used to construct linkage maps. Thus, only a small portion of SNPs were placed on the linkage maps. Nonetheless, more markers were placed on the linkage maps in this study than microsatellites in our previous QTL mapping studies (Vallejo et al., 2014a,b).

\section{SNPs Associated with BCWD Resistance and Spleen Size based on GWAS}

Among 7,595 SNPs assigned to 29 chromosomes of rainbow trout, 5,220 SNPs had genotype call rates over $70 \%$ in the combined mapping families 2012473 and 2012474, and were used for GWAS to identify SNPs associated BCWD resistance. Of the 5,220 SNPs analyzed, 18 SNPs associated with BCWD resistance were identified (Figures 1 and 2, Table 1). As expected, three SNPs on chromosome Omy19 were associated with BCWD resistance. Surprisingly, seven SNPs on chromosome Omy8 were also associated with BCWD resistance. The other eight significant SNPs for BCWD resistance were located on chromosomes Omy2, Omy15, Omy22, Omy26, and Omy28 with only one to three significant SNPs on each chromosome.

Among 5,478 SNPs with genotype call rates over 70\% in the mapping family 2012474, 20 SNPs associated with spleen index were identified (Figure 3, Table 2). Eleven of these 20 SNPs were located on chromosome Omy5, and the other nine SNPs 


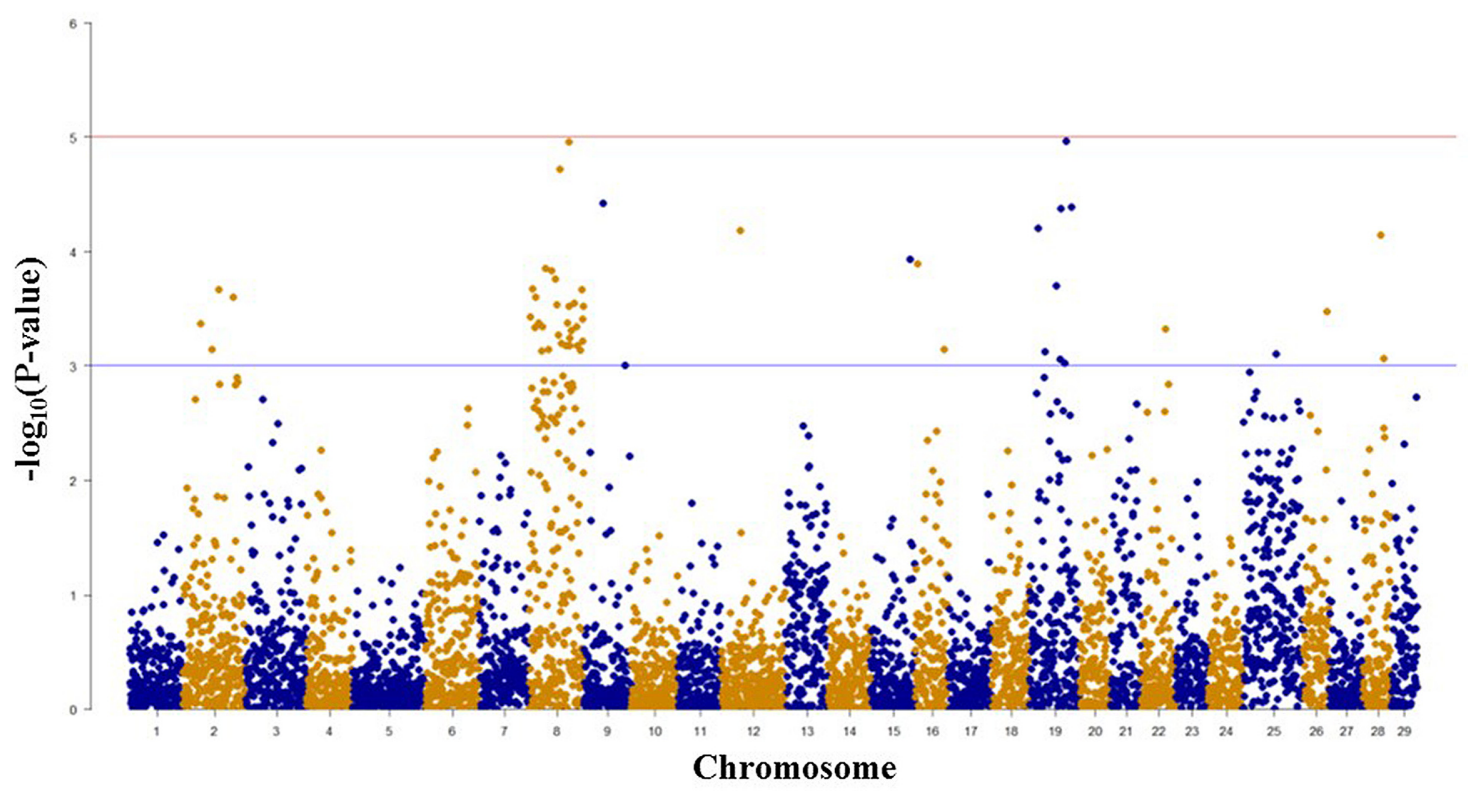

FIGURE 1 | Results of genome-wide association studies of survival status using GenABEL's GRAMMAR-Gamma method. The horizontal lines represent $P=1 \times 10^{-3}$ and $P=1 \times 10^{-5}$, respectively.

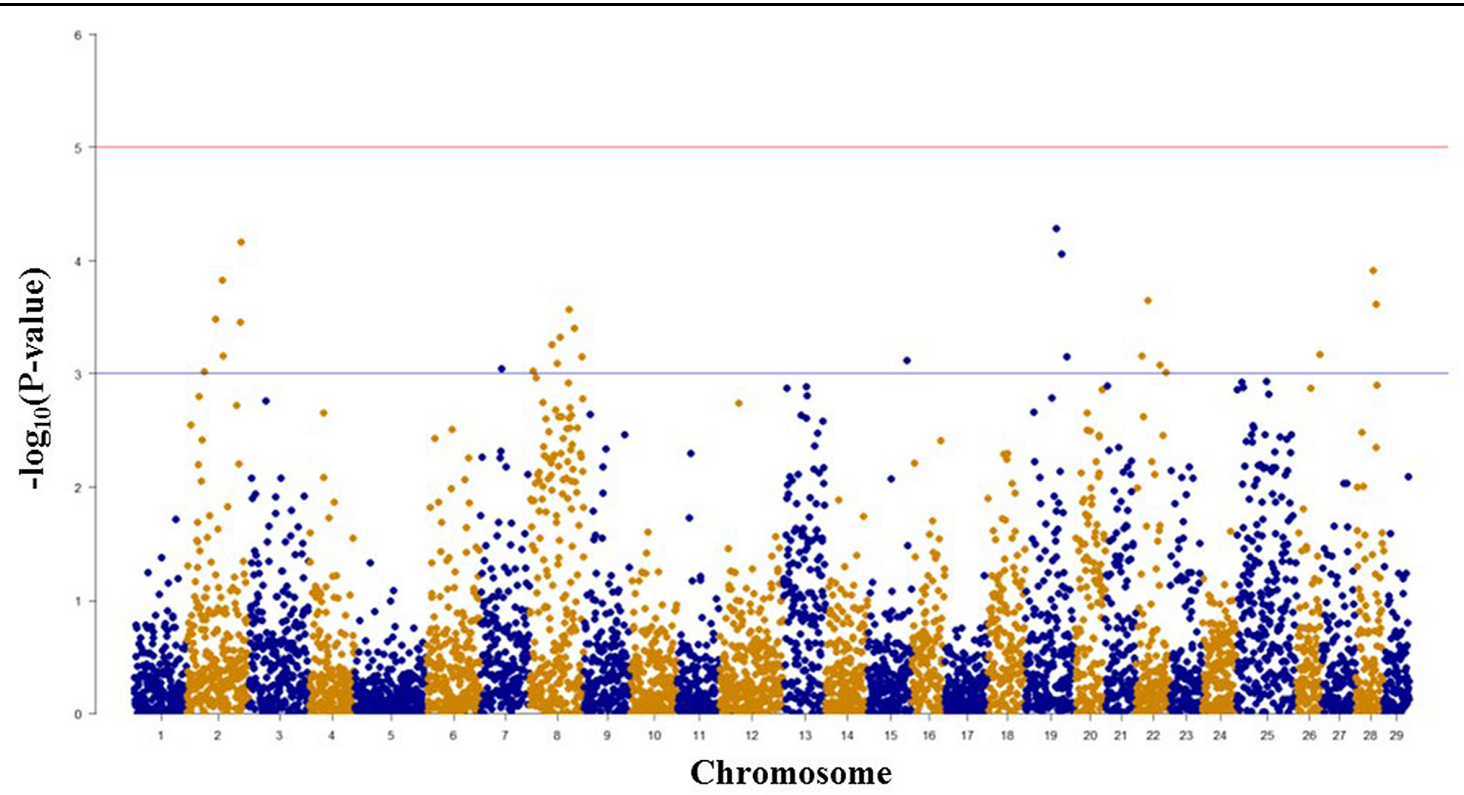

FIGURE 2 | Results of genome-wide association studies of survival days using GenABEL's GRAMMAR-Gamma method. The horizontal lines represent $P=1 \times 10^{-3}$ and $P=1 \times 10^{-5}$, respectively.

were located on chromosomes Omy4, Omy12, Omy23, Omy24, Omy27, and Omy29 (OmySex) with one to three significant SNPs on each chromosome.

\section{Linkage-based QTL Mapping for BCWD Resistance and Spleen Size}

Quantitative trait loci mapping using the half-sib module of GridQTL revealed three significant QTL for BCWD resistance
(Table 3). These three significant QTL were located on chromosomes Omy8, Omy19, and Omy25, and they explained 72\% (STATUS; Sire), 29\% (STATUS; Dam), and 34\% (DAYS; Sire) of the phenotypic variance, respectively. Consistent with our previous report (Vallejo et al., 2014b), the QTL on Omy19 was derived from the dam 2008132002. The QTL on chromosomes Omy8 and Omy25 were derived from the sires. Suggestive QTL for BCWD resistance were identified on chromosomes Omy2, 


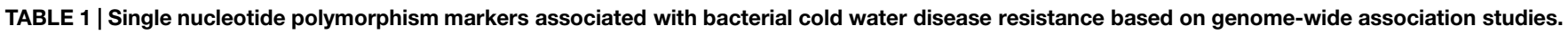

\begin{tabular}{|c|c|c|c|c|c|c|c|}
\hline \multirow[b]{2}{*}{ SNP } & \multirow[b]{2}{*}{ Chromosome } & \multirow[b]{2}{*}{ Allele 1} & \multirow[b]{2}{*}{ Allele 2} & \multicolumn{2}{|c|}{ Survival status } & \multicolumn{2}{|c|}{ Survival days } \\
\hline & & & & Effect $^{a}$ & $P$ & Effect $^{a}$ & $P$ \\
\hline RTRAD89nt118565 & 2 & $\mathrm{~T}$ & C & -0.36 & $2.15 \mathrm{E}-04$ & -4.95 & 1.49E-04 \\
\hline RTRAD89nt095147 & 2 & A & $\mathrm{G}$ & -0.34 & 4.30E-04 & -4.29 & 9.68E-04 \\
\hline RTRAD89nt110567 & 2 & G & $\mathrm{T}$ & -0.33 & 7.24E-04 & -4.68 & 3.31E-04 \\
\hline RTRAD89nt131357 & 8 & C & $\mathrm{T}$ & 0.33 & 1.10E-05 & 3.70 & 2.72E-04 \\
\hline RTRAD89nt116978 & 8 & $\mathrm{G}$ & C & 0.40 & $1.93 \mathrm{E}-05$ & 4.37 & 4.77E-04 \\
\hline RTRAD89nt103341 & 8 & $\mathrm{~T}$ & C & 0.45 & 1.47E-04 & 5.44 & 5.53E-04 \\
\hline RTRAD89nt078368 & 8 & $A$ & $\mathrm{C}$ & 0.42 & $2.15 \mathrm{E}-04$ & 5.07 & 9.48E-04 \\
\hline RTRAD89nt148358 & 8 & C & $\mathrm{T}$ & 0.36 & $2.17 \mathrm{E}-04$ & 4.43 & 7.16E-04 \\
\hline RTRAD89nt140425 & 8 & A & G & 0.44 & 2.87E-04 & 5.72 & 3.98E-04 \\
\hline RTRAD89nt112550 & 8 & $\mathrm{G}$ & $\mathrm{T}$ & 0.42 & 2.92E-04 & 5.23 & 8.18E-04 \\
\hline RTRAD89nt139370 & 15 & $\mathrm{G}$ & C & 0.38 & $1.17 \mathrm{E}-04$ & 4.50 & 7.63E-04 \\
\hline RTRAD89nt135689 & 19 & C & $\mathrm{T}$ & 0.43 & 1.09E-05 & 5.09 & 8.76E-05 \\
\hline RTRAD89nt141241 & 19 & G & $A$ & -0.40 & 4.13E-05 & -4.39 & 7.07E-04 \\
\hline RTRAD89nt125108 & 19 & $\mathrm{G}$ & $A$ & 0.40 & $4.21 \mathrm{E}-05$ & 5.29 & $5.22 \mathrm{E}-05$ \\
\hline RTRAD89nt127892 & 22 & $\mathrm{~T}$ & C & -0.23 & 4.78E-04 & -3.03 & $8.41 \mathrm{E}-04$ \\
\hline RTRAD89nt142090 & 26 & $G$ & A & -0.30 & 3.36E-04 & -3.83 & 6.82E-04 \\
\hline RTRAD89nt128385 & 28 & $\mathrm{G}$ & $A$ & -0.46 & 7.26E-05 & -5.93 & 1.23E-04 \\
\hline RTRAD89nt137710 & 28 & C & $\mathrm{T}$ & -0.26 & 8.68E-04 & -3.77 & 2.43E-04 \\
\hline
\end{tabular}

${ }^{a}$ Effect of allele 2 on BCWD resistance phenotypes. Negative value means that allele 1 is associated with BCWD resistance.

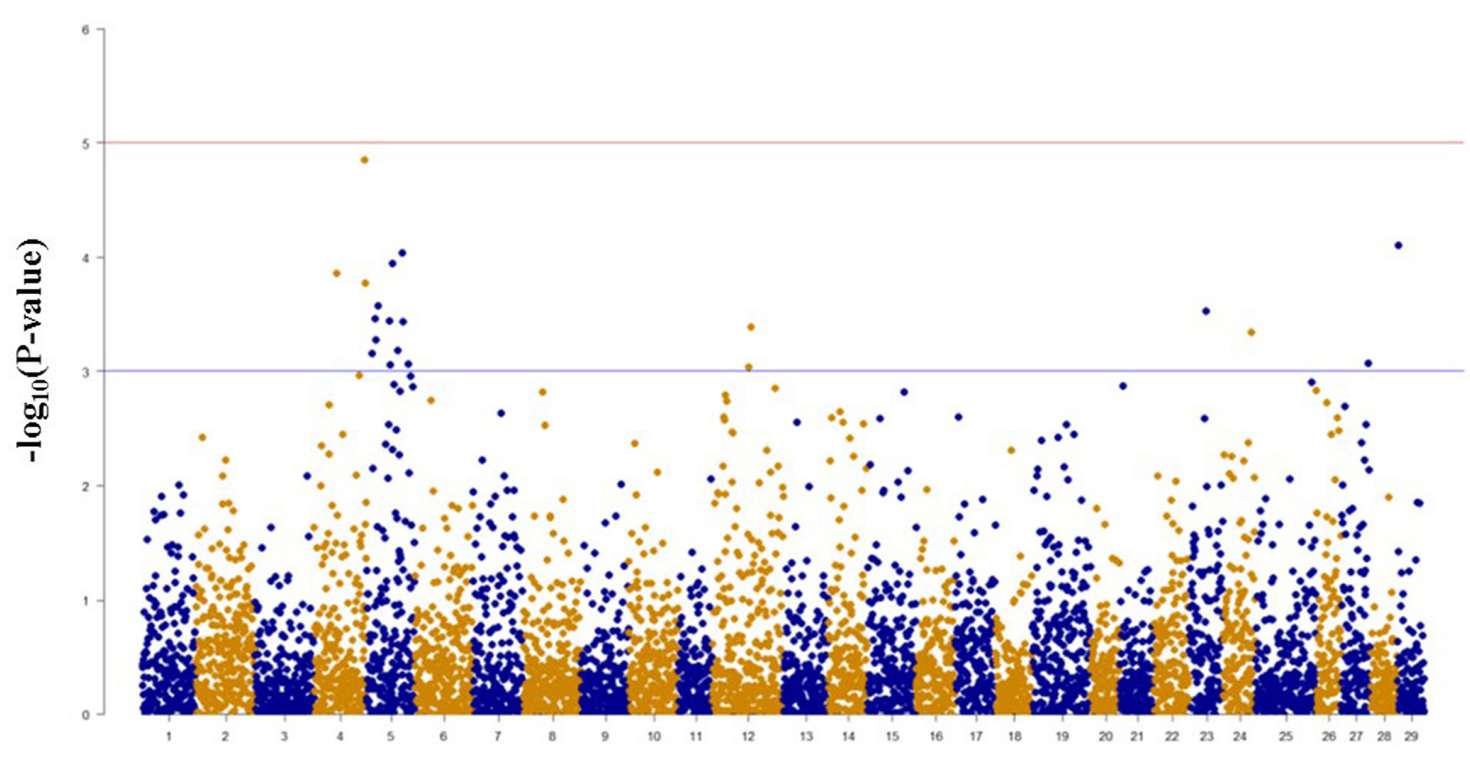

Chromosome

FIGURE 3 | Results of genome-wide association studies of spleen index using GenABEL's GRAMMAR-Gamma method. The horizontal lines represent $P=1 \times 10^{-3}$ and $P=1 \times 10^{-5}$, respectively.

Omy13, Omy16, Omy20, Omy26, and Omy28 (Table 3). Only one significant QTL for spleen index was identified (Table 4), which was located on chromosome Omy2. This significant spleen index QTL explained 63\% of the spleen index variation. Eight suggestive QTL for spleen index were also identified in this study (Table 4) including a suggestive QTL on Omy19 inherited from the sire.

\section{Discussion}

Previously, we used seven microsatellites to validate the QTL for BCWD resistance and for spleen index on chromosome Omy19 in two rainbow trout half-sib families (Vallejo et al., 2014b). In this study, we conducted a whole-genome scan in the same two half-sib families using RAD sequencing to identify 
TABLE 2 | SNPs associated with spleen index based on GWAS.

\begin{tabular}{|c|c|c|c|c|c|}
\hline SNP & Chromosome & Allele 1 & Allele 2 & Effect $^{a}$ & $P$ \\
\hline RTRAD89nt147353 & 4 & $G$ & A & -0.97 & $1.42 \mathrm{E}-05$ \\
\hline RTRAD89nt107990 & 4 & G & $\mathrm{T}$ & -0.79 & 1.40E-04 \\
\hline RTRAD89nt148293 & 4 & G & $A$ & -0.82 & 1.69E-04 \\
\hline RTRAD89nt134509 & 5 & $A$ & $G$ & 0.85 & $9.21 \mathrm{E}-05$ \\
\hline RTRAD89nt114301 & 5 & $\mathrm{G}$ & $\mathrm{T}$ & 0.62 & 1.14E-04 \\
\hline RTRAD89nt091881 & 5 & $\mathrm{~T}$ & C & 0.80 & 2.69E-04 \\
\hline RTRAD89nt085023 & 5 & C & $\mathrm{T}$ & 0.78 & 3.47E-04 \\
\hline RTRAD89nt109036 & 5 & $\mathrm{~T}$ & $\mathrm{G}$ & 0.78 & 3.63E-04 \\
\hline RTRAD89nt137156 & 5 & $A$ & $\mathrm{G}$ & -0.79 & 3.66E-04 \\
\hline RTRAD89nt089304 & 5 & $A$ & $\mathrm{G}$ & 0.81 & 5.31E-04 \\
\hline RTRAD89nt127451 & 5 & $\mathrm{G}$ & $\mathrm{T}$ & 0.80 & 6.62E-04 \\
\hline RTRAD89nt081765 & 5 & C & A & -0.74 & 6.99E-04 \\
\hline RTRAD89nt142812 & 5 & C & $\mathrm{T}$ & 0.52 & 8.70E-04 \\
\hline RTRAD89nt109928 & 5 & $\mathrm{~T}$ & C & 0.74 & 8.82E-04 \\
\hline RTRAD89nt112119 & 12 & C & A & -0.75 & 4.09E-04 \\
\hline RTRAD89nt110052 & 12 & A & $\mathrm{T}$ & 0.52 & $9.22 \mathrm{E}-04$ \\
\hline RTRAD89nt109171 & 23 & $\mathrm{~T}$ & $\mathrm{C}$ & -0.50 & 2.99E-04 \\
\hline RTRAD89nt140132 & 24 & $\mathrm{G}$ & A & 0.81 & 4.59E-04 \\
\hline RTRAD89nt142053 & 27 & $\mathrm{~T}$ & $\mathrm{C}$ & 0.47 & 8.56E-04 \\
\hline RTRAD89nt072289 & 29 & C & $\mathrm{T}$ & 0.98 & 7.88E-05 \\
\hline
\end{tabular}

aEffect of allele 2 on spleen index. Negative value means that allele 1 is associated with higher spleen index.

SNPs associated with the same two traits. Based on GWAS, 18 SNPs associated with BCWD resistance and 20 SNPs associated with spleen index were identified. Linkage-based QTL mapping revealed three significant QTL for BCWD resistance and one significant QTL for spleen index.

\section{Consistency between the Results of GWAS and Linkage-based QTL Mapping}

We used both GWAS and linkage-based QTL mapping to identify SNP markers associated with BCWD resistance or spleen index in this study. Overall, the results between these two approaches were consistent, especially between significant BCWD QTL and chromosomes with three or more significant SNPs identified by GWAS. There were some discrepancies between GWAS and linkage-based QTL mapping for regions that contained only one or two significant SNPs (Tables 1 and 2), and we suggest caution in interpretation of results from these regions. Based on GWAS, there were three chromosomes Omy2, Omy8, and Omy19 with three or more significant SNPs for BCWD resistance. Consistent with this result, linkage-based QTL mapping revealed significant QTL for BCWD resistance on chromosomes Omy8 and Omy19, and a suggestive QTL for BCWD resistance on chromosome Omy2. However, we did not identify SNPs associated with BCWD resistance on chromosome 25 based on GWAS. Thus, we suggest caution for the BCWD QTL on chromosome Omy25.

The consistency between GWAS and linkage-based QTL mapping for spleen index is not as clear as that for BCWD resistance. This might be because only 96 fish were used for spleen index in this study. The only significant QTL for spleen index identified in this study was located on chromosome Omy2, and RTRAD89nt119684 was near the peak of the QTL plot. This SNP can only be assigned to chromosome Omy2 using the combined genotype data of mapping families 2012473 and 2012474. Thus, it was excluded from GWAS analysis for spleen index in family 2012474. Based on GWAS, two chromosomes Omy4 and Omy5 had three and 11 significant SNPs for spleen

TABLE 3 | Quantitative trait loci for BCWD resistance identified by half-sib QTL mapping.

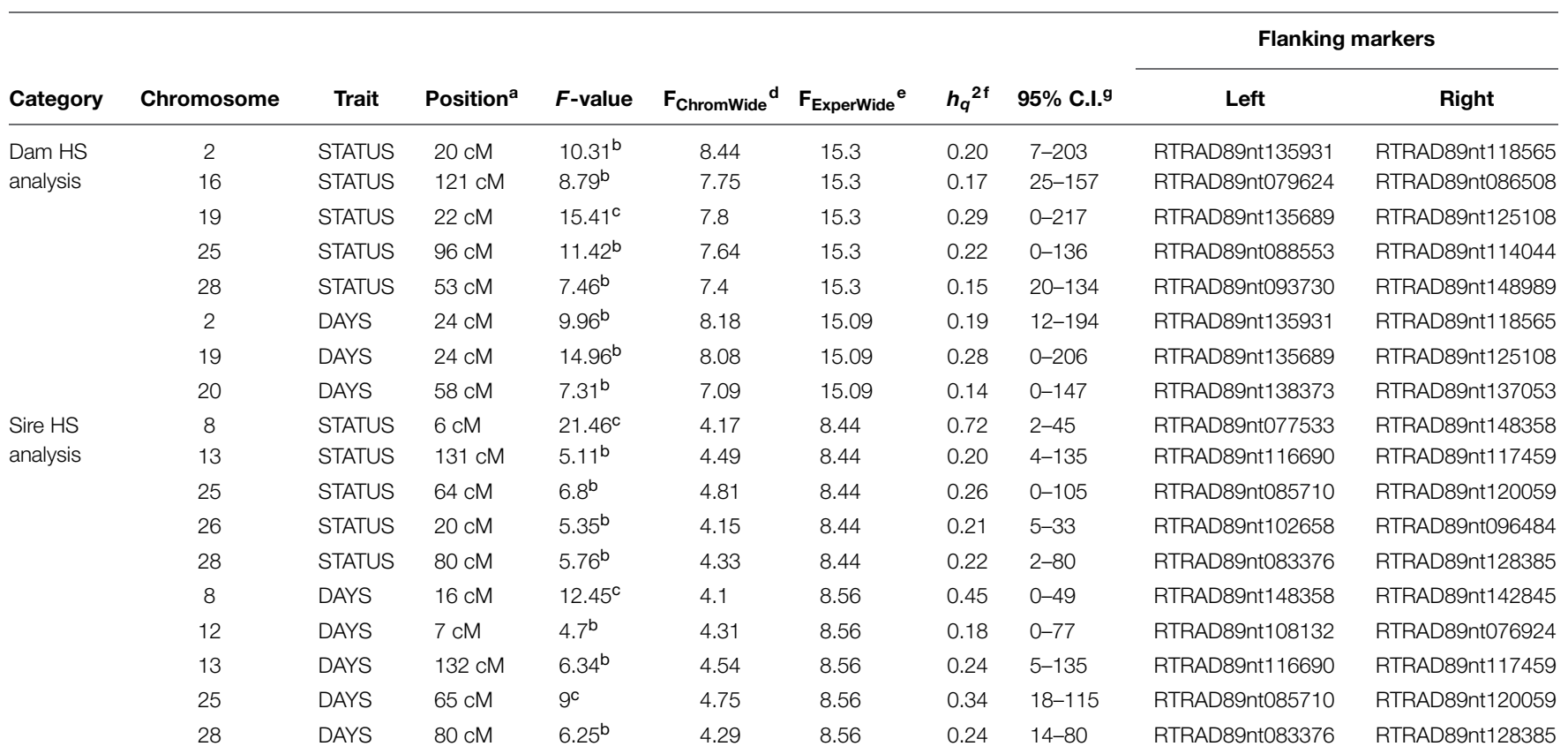

${ }^{\mathrm{a}}$ The peak position of QTL plot; ${ }^{\mathrm{b}}$ suggestive QTL; ${ }^{\mathrm{c}}$ significant $\mathrm{QTL}$; ${ }^{\mathrm{d}}$ F-value threshold for chromosome-wide significance; ${ }^{\mathrm{e}}$ F-value threshold for experiment-wide significance; ${ }^{\mathrm{f}}$ phenotypic variance explained by each QTL; $995 \%$ confidence interval. 
TABLE 4 | Quantitative trait loci for spleen index identified by half-sib QTL mapping.

\begin{tabular}{|c|c|c|c|c|c|c|c|c|c|}
\hline \multirow[b]{2}{*}{ Category } & \multirow[b]{2}{*}{ Chromosome } & \multirow[b]{2}{*}{ Position ${ }^{a}$} & \multirow[b]{2}{*}{$F$-value } & \multirow[b]{2}{*}{ F $_{\text {ChromWide }}{ }^{d}$} & \multirow[b]{2}{*}{$F_{\text {ExperWide }}{ }^{e}$} & \multirow[b]{2}{*}{$h_{q}^{2 f}$} & \multirow[b]{2}{*}{$95 \%$ C.I. ${ }^{g}$} & \multicolumn{2}{|c|}{ Flanking markers } \\
\hline & & & & & & & & Left & Right \\
\hline \multirow[t]{4}{*}{ Dam HS analysis } & 5 & $3 \mathrm{cM}$ & $11.36^{b}$ & 7.7 & 14.64 & 0.43 & $3-194$ & RTRAD89nt108265 & RTRAD89nt137156 \\
\hline & 7 & $130 \mathrm{cM}$ & $8.54^{b}$ & 8.14 & 14.64 & 0.33 & $12-215$ & RTRAD89nt136795 & RTRAD89nt071431 \\
\hline & 26 & 152 cM & $8.67^{b}$ & 7.39 & 14.64 & 0.34 & $19-152$ & RTRAD89nt099530 & RTRAD89nt148730 \\
\hline & 27 & $22 \mathrm{cM}$ & $7.82^{b}$ & 7.25 & 14.64 & 0.31 & $14-142$ & RTRAD89nt113238 & RTRAD89nt132122 \\
\hline \multirow[t]{5}{*}{ Sire HS analysis } & 2 & $6 \mathrm{cM}$ & $17.6^{\mathrm{c}}$ & 6.59 & 13.1 & 0.63 & $0-36$ & RTRAD89nt119684 & RTRAD89nt149352 \\
\hline & 6 & $22 \mathrm{cM}$ & $9.28^{\mathrm{b}}$ & 5.95 & 13.1 & 0.36 & $0-59$ & RTRAD89nt089147 & RTRAD89nt077857 \\
\hline & 17 & $52 \mathrm{cM}$ & $8.79^{b}$ & 6.71 & 13.1 & 0.34 & $36-146$ & RTRAD89nt074950 & RTRAD89nt090428 \\
\hline & 19 & $27 \mathrm{cM}$ & $8.88^{b}$ & 5.63 & 13.1 & 0.35 & $22-62$ & RTRAD89nt094659 & RTRAD89nt149131 \\
\hline & 22 & $96 \mathrm{cM}$ & $7.36^{b}$ & 6.53 & 13.1 & 0.29 & $21-96$ & RTRAD89nt138842 & RTRAD89nt109237 \\
\hline
\end{tabular}

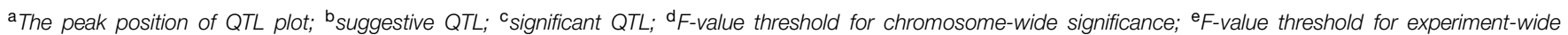
significance; ${ }^{f}$ phenotypic variance explained by each QTL; ${ }^{9} 95 \%$ confidence interval.

index, respectively. Similar to this result, a suggestive QTL for spleen index was identified on chromosome Omy5. The QTL plot for spleen index peaked with an $F$-value 7.38 on chromosome Omy4, which was almost near the threshold $F$-value 7.43 for a suggestive QTL.

The consistency between GWAS and linkage-based QTL mapping is not totally surprising because samples from two mapping families were used for GWAS in this study. Ideally, large number of unrelated animals should be used for GWAS. In reality, samples for GWAS are often collected from family based selective breeding program. In that case, we suggest using higher number of families and fewer progeny per family. The number of SNPs used for GWAS in this study is also not ideal. Recently, a rainbow trout 57K SNP chip has been developed (Palti et al., 2015a), which provides a better tool for GWAS in rainbow trout.

\section{The Significant BCWD QTL on Chromosome Omy19}

Using microsatellites for QTL mapping, a significant BCWD QTL on Omy19 was initially identified in the mapping family 2008132 (Wiens et al., 2013b), and this QTL was validated in a subsequent generation (Vallejo et al., 2014b). As expected, this QTL was validated again using RAD-seq for genotyping in this study. Therefore, this QTL is robust and is a suitable target for fine mapping and identification of positional candidate genes for BCWD resistance.

It is challenging to identify positional candidate genes for the BCWD QTL on chromosome Omy19 using the current draft genome sequences of rainbow trout. Based on GWAS, three SNPs (RTRAD89nt125108, RTRAD89nt135689, and RTRAD89nt141241) on chromosome Omy19 (Table 1) were associated with BCWD resistance in this study. Markers RTRAD89nt125108 and RTRAD89nt135689 flank the peak of the QTL plot of chromosome Omy19. We used the sequences of these three SNPs to query the draft sequences of rainbow trout deposited at http://www.animalgenome.org/repository/ aquaculture/ (We refer to this website as animalgenome.org from now on). RTRAD89nt125108 was mapped onto four BAC (bacteria artificial chromosome) sequences, two perfect sequence matches for each SNP allele. Likewise, RTRAD89nt135689 was mapped onto two BAC sequences, one perfect sequence match for each allele of the SNP. RTRAD89nt141241 was mapped to three BAC sequences. Each of these three SNPs was not only mapped to multiple BAC sequences but also was located on at least two BAC physical contigs. For example, the two BAC clones having perfect match with the sequence of allele A of RTRAD89nt125108 were located on BAC physical contig 11226 (Palti et al., 2012). One of the two BACs matching perfectly with the sequence of allele $G$ of RTRAD89nt125108 was located on BAC physical contig 11227 (Palti et al., 2012), the other BAC clone could not be assigned to its BAC physical contig because the full name of the BAC clone was unknown (BACs were sequenced in pools and some sequences could not be traced back to its original BAC clone due to technical challenges). The mapping of each SNP allele to a separate BAC physical contig is not surprising because of the well documented homeology between the terminal ends of chromosomes Omy19 and Omy10 in rainbow trout (Phillips et al., 2006; Rexroad et al., 2008). Previously we have shown that the BCWD QTL on Omy19 is near the end of chromosome and that most microsatellites near the QTL are mapped to both chromosomes Omy19 and Omy10 (Wiens et al., 2013b). More research is needed to determine which BAC sequences are likely on chromosome Omy19 and which BAC sequences belong to Omy10. We also used the sequences of the three SNPs to query the scaffold sequences of rainbow trout recently published by Berthelot et al. (2014). Each SNP identified one positive scaffold (Table 5). However, all three scaffold sequences were less than $40 \mathrm{~kb}$ and were located on unknown chromosomes of rainbow trout. Hence, improvement of the genome sequence for this QTL region is needed to identify positional candidate genes for this QTL.

\section{Additional Significant QTL for BCWD Resistance}

In addition to the significant BCWD QTL on chromosome Omy19, two significant BCWD QTL derived from the sires were detected in this study. They were located on chromosomes Omy8 and Omy25, respectively. Both sires used in this study were derived from the mapping family 2009044, and a significant BCWD QTL on chromosome Omy8 and a suggestive BCWD 
TABLE 5 | Rainbow trout genome scaffolds (Berthelot et al., 2014) matched by SNPs associated with BCWD resistance on chromosomes Omy8 and Omy19.

\begin{tabular}{lcll}
\hline SNP & Chromosome & Scaffold & Size (bP) \\
\hline RTRAD89nt131357 & 8 & scaffold_8633 & 26559 \\
RTRAD89nt078368 & 8 & scaffold_450 & 800741 \\
RTRAD89nt148358 & 8 & scaffold_262 & 1102120 \\
RTRAD89nt140425 & 8 & scaffold_7501 & 31078 \\
RTRAD89nt116978 & 8 & scaffold_6507 & 36069 \\
RTRAD89nt103341 & 8 & scaffold_45159 & 3029 \\
RTRAD89nt135689 & 19 & scaffold_14916 & 12228 \\
RTRAD89nt141241 & 19 & scaffold_6543 & 35825 \\
RTRAD89nt125108 & 19 & scaffold_6016 & 39141 \\
\hline
\end{tabular}

a Single nucleotide polymorphism marker RM20090703790 associated with BCWD resistance in mapping family 2009070 (Palti et al., 2015b) was also located on this scaffold. But, these two SNPs are $713 \mathrm{~Kb}$ apart on this scaffold.

QTL on chromosome Omy25 were reported in our previous study using microsatellites for QTL mapping (Vallejo et al., 2014a). The QTL on chromosome Omy8 explained a large proportion of phenotypic variance in this study. However, it is known that variance explained by QTL could be over-estimated in mapping populations. The QTL effects should be further evaluated in breeding populations. Mapping families 2009044 and 2009070 share a pair of grandparents. A significant QTL for BCWD resistance on chromosome Omy8 was also detected in mapping family 2009070 in our previous study (Vallejo et al., 2014a) and this QTL was validated using RAD-seq in a recent study (Palti et al., 2015b). Moreover, among the 12 SNPs associated with BCWD resistance reported by Campbell et al. (2014), SNP R46637 is also located on chromosome Omy8. We do not know whether R46637 is located in the chromosome Omy8 BCWD QTL region reported in this study. Nonetheless, the significant BCWD QTL on chromosome Omy8 deserve more attentions in the future.

Seven SNPs associated with the BCWD QTL on chromosome Omy8 were identified in this study. We used the seven SNP sequences to query the rainbow trout genome sequences deposited at animalgenome.org. Three SNPs were mapped onto three different BAC clone sequences, respectively. SNP RTRAD89nt148358 was mapped onto three BAC sequences. The other three SNPs had no significant match with the BAC sequences. Among the seven SNPs associated with the BCWD QTL on chromosome Omy8, only one SNP had no match with the rainbow trout scaffold sequences published by Berthelot et al. (2014). The other six SNPs were mapped onto six different scaffold sequences with a total length near $2 \mathrm{Mb}$ (Table 5). Thus, improved reference genome sequence and additional mapping studies are needed to identify positional candidate genes for this QTL.

\section{Non-Pleiotropic QTL for BCWD Resistance and Spleen Index on Chromosome Omy19 of Rainbow Trout}

Consistent with the association between BCWD resistance and spleen size (Hadidi et al., 2008), both QTL for BCWD resistance and spleen index on chromosome Omy19 were identified in mapping family 2008132 (Wiens et al., 2013b). However, two separately inherited QTL for BCWD resistance and spleen index on chromosome Omy19 were identified in four additional mapping families we genotyped with seven microsatellites (Vallejo et al., 2014b). In this study, we used RAD-seq for genotyping and 7,849 informative SNPs were identified to further resolve inheritance of these two QTL. Based on GWAS, three SNPs on chromosome Omy19 were significantly associated with BCWD resistance, but there was no significant SNP for spleen index on chromosome Omy19. Similarly, half-sib QTL mapping revealed a significant QTL for BCWD resistance on chromosome Omy19 of fish 2008132002, and there was no spleen index QTL on chromosome Omy19 of fish 2008132002. Therefore, we independently validated our microsatellite-based finding that non-pleiotropic QTL control BCWD resistance and spleen index on chromosome Omy19.

The conclusion of non-pleiotropic QTL controlling BCWD resistance and spleen index on chromosome Omy19 is also supported by the identification of a suggestive QTL for spleen index derived from the sire 2009044044 but no corresponding QTL for BCWD resistance on this chromosome. Moreover, our results also indicated that the non-pleiotropic QTL for BCWD resistance and spleen index might also be extended to other chromosomes. Among the 18 SNPs associated with BCWD resistance and 20 SNPs associated with spleen index identified in this study, none of them was significant for both traits. Although some of the QTL for these two traits were located on the same chromosomes in this study, they were derived from different parents. For example, a significant QTL for spleen index derived from the sire 2009044044 was identified on chromosome Omy2, but the suggestive QTL for BCWD resistance was derived from the dam 2008132002.

The non-pleiotropic relationship between BCWD resistance and spleen index is also consistent with our follow-up studies. Recently, Wiens et al. (2015) reported that reduction of spleen size by splenectomy did not alter BCWD resistance in rainbow trout and thus the greater spleen size in some families does not directly influence resistance, rather it is a non-pleiotropic correlation. The lack of a significant genetic correlation between BCWD resistance and spleen index in our odd-year spawning line of rainbow trout in contrast to the even-year spawning line indicates that the association of these two traits across genetic populations is inconsistent and complex (Wiens et al., 2013b). Given the variable and non-pleiotropic association between these two traits, for future studies, we recommend focusing on mapping the QTL for BCWD post-challenge survival instead of spleen index as the most productive direction for understanding the genetic control of BCWD resistance in rainbow trout.

\section{Conclusion}

Previously, we used seven microsatellites to validate the QTL for BCWD resistance and for spleen index on chromosome Omy19 (Vallejo et al., 2014b). In this study we conducted a wholegenome scan in two half-sib rainbow trout mapping families with 
the aim to identify SNPs associated with BCWD resistance and spleen size using both GWAS and linkage-based QTL mapping approaches. Among 7,849 SNPs identified in this study, 18 SNPs associated with BCWD resistance were identified by GWAS. In addition to the previously identified dam-derived $\mathrm{BCWD}$ resistance QTL on chromosome Omy19 (Wiens et al., 2013b; Vallejo et al., 2014b), we identified two new sire-derived BCWD QTL on chromosomes Omy8 and Omy25, respectively. GWAS analysis also revealed 20 SNPs associated with spleen size. Despite the significant increase of genome coverage in this study, none of these 20 SNPs was located on chromosome Omy19. Furthermore, half-sib QTL mapping did not reveal dam-derived QTL for spleen index on chromosome Omy19 either. These results confirm that BCWD resistance and spleen index are not controlled by pleiotropic QTL on chromosome Omy19. Thus, we should focus on QTL for BCWD resistance instead of spleen size in the future. To facilitate marker-assisted selection for BCWD resistance, we are in the process to validate the SNPs reported in this study in the breeding populations of rainbow trout. The SNP markers reported in this study will also facilitate fine mapping to identify positional candidate genes for BCWD resistance in rainbow trout.

\section{References}

Aulchenko, Y. S., Ripke, S., Isaacs, A., and Van Duijn, C. M. (2007). GenABEL: an $\mathrm{R}$ library for genorne-wide association analysis. Bioinformatics 23, 1294-1296. doi: 10.1093/bioinformatics/btm108

Baird, N. A., Etter, P. D., Atwood, T. S., Currey, M. C., Shiver, A. L., Lewis, Z. A., et al. (2008). Rapid SNP discovery and genetic mapping using sequenced RAD markers. PLOS ONE 3:e3376. doi: 10.1371/journal.pone. 0003376

Berthelot, C., Brunet, F., Chalopin, D., Juanchich, A., Bernard, M., Noel, B., et al. (2014). The rainbow trout genome provides novel insights into evolution after whole-genome duplication in vertebrates. Nat. Commun. 5, 3657. doi: $10.1038 /$ ncomms 4657

Campbell, N. R., Lapatra, S. E., Overturf, K., Towner, R., and Narum, S. R. (2014). Association mapping of disease resistance traits in rainbow trout using restriction site associated DNA sequencing. G3 (Bethesda) 4, 2473-2481. doi: $10.1534 / \mathrm{g} 3.114 .014621$

Hadidi, S., Glenney, G. W., Welch, T. J., Silverstein, J. T., and Wiens, G. D. (2008). Spleen size predicts resistance of rainbow trout to Flavobacterium psychrophilum challenge. J. Immunol. 180, 4156-4165. doi: 10.4049/jimmunol.180.6.4156

Leeds, T. D., Silverstein, J. T., Weber, G. M., Vallejo, R. L., Palti, Y., Rexroad, C. E., et al. (2010). Response to selection for bacterial cold water disease resistance in rainbow trout. J. Anim. Sci. 88, 1936-1946. doi: 10.2527/jas.200 9-2538

Liu, S., Vallejo, R. L., Gao, G., Palti, Y., Weber, G. M., Hernandez, A., et al. (2015). Identification of single-nucleotide polymorphism markers associated with cortisol response to crowding in rainbow trout. Mar. Biotechnol. (NY) 17, 328-337. doi: 10.1007/s10126-015-9621-4

Loch, T. P., and Faisal, M. (2015). Emerging flavobacterial infections in fish: a review. J. Adv. Res. 6, 283-300. doi: 10.1016/j.jare.2014. 10.009

Matise, T. C., Perlin, M., and Chakravarti, A. (1994). Automated construction of genetic-linkage maps using an expert-system (multimap) - a human genome linkage map. Nat. Genet. 6, 384-390. doi: 10.1038/ng0494-384

Miller, M. R., Dunham, J. P., Amores, A., Cresko, W. A., and Johnson, E. A. (2007). Rapid and cost-effective polymorphism identification and genotyping using restriction site associated DNA (RAD) markers. Genome Res. 17, 240-248. doi: $10.1101 /$ gr.5681207

\section{Acknowledgments}

We would like to thank Roseanna Long and Kristy Shewbridge for making the RAD libraries, and Joel Caren and Travis Moreland for care of fish used in this study. This work was supported by Agricultural Research Service CRIS Project 193032000-006 "Integrated Research to Improve On-Farm Animal Health in Salmonid Aquaculture", and CRIS Project 193031000-012 "Integrated Research Approaches for Improving Production Efficiency in Salmonids". Mention of trade names or commercial products in this publication is solely for the purpose of providing specific information and does not imply recommendation or endorsement by the U.S. Department of Agriculture (USDA). USDA is an equal opportunity provider and employer.

\section{Supplementary Material}

The Supplementary Material for this article can be found online at: http://journal.frontiersin.org/article/10.3389/fgene. 2015.00298

Nematollahi, A., Decostere, A., Pasmans, F., and Haesebrouck, F. (2003). Flavobacterium psychrophilum infections in salmonid fish. J. Fish Dis. 26, 563-574. doi: 10.1046/j.1365-2761.2003.00488.x

Palti, Y., Gao, G., Liu, S., Kent, M. P., Lien, S., Miller, M. R., et al. (2015a). The development and characterization of a $57 \mathrm{~K}$ single nucleotide polymorphism array for rainbow trout. Mol. Ecol. Resour. 15, 662-672. doi: 10.1111/17550998.12337

Palti, Y., Vallejo, R. L., Gao, G., Liu, S., Hernandez, A. G., Rexroad, C. E. III., et al. (2015b). Detection and validation of QTL affecting bacterial cold water disease resistance in rainbow trout using restriction-site associated DNA sequencing. PLoS ONE 10:e0138435. doi: 10.1371/journal.pone.0138435

Palti, Y., Gao, G., Miller, M. R., Vallejo, R. L., Wheeler, P. A., Quillet, E., et al. (2014). A resource of single-nucleotide polymorphisms for rainbow trout generated by restriction-site associated DNA sequencing of doubled haploids. Mol. Ecol. Resour. 14, 588-596. doi: 10.1111/1755-0998.12204

Palti, Y., Genet, C., Gao, G. T., Hu, Y. Q., You, F. M., Boussaha, M., et al. (2012). A second generation integrated map of the rainbow trout (Oncorhynchus mykiss) genome: analysis of conserved synteny with model fish genomes. Mar. Biotechnol. 14, 343-357. doi: 10.1007/s10126-0119418-Z

Phillips, R., Nichols, K., Dekoning, J., Morasch, M., Keatley, K., Rexroad, C., et al. (2006). Assignment of rainbow trout linkage groups to specific chromosomes. Genetics 174, 1661-1670. doi: 10.1534/genetics.105.055269

Rexroad, C. E. III, Palti, Y., Gahr, S. A., and Vallejo, R. L. (2008). A second generation genetic map for rainbow trout (Oncorhynchus mykiss). BMC Genet. 9:74. doi: 10.1186/1471-2156-9-74

Seaton, G., Hernandez, J., Grunchec, J. A., White, I., Allen, J., De Koning, D. J., et al. (2006). "GridQTL: a grid portal for QTL mapping of compute intensive datasets," in Proceedings of the 8th World Congress on Genetics Applied to Livestock Production, Belo Horizonte.

Starliper, C. E. (2011). Bacterial coldwater disease of fishes caused by Flavobacterium psychrophilum. J. Adv. Res. 2, 97-108. doi: 10.1016/j.jare.2010.04.001

Svishcheva, G. R., Axenovich, T. I., Belonogova, N. M., Van Duijn, C. M., and Aulchenko, Y. S. (2012). Rapid variance components-based method for whole-genome association analysis. Nat. Genet. 44, 1166-1170. doi: 10.1038/ ng. 2410

Vallejo, R. L., Palti, Y., Liu, S. X., Evenhuis, J. P., Gao, G. T., Rexroad, C. E., et al. (2014a). Detection of QTL in rainbow trout affecting survival when 
challenged with Flavobacterium psychrophilum. Mar. Biotechnol. 16, 349-360. doi: 10.1007/s10126-013-9553-9

Vallejo, R. L., Palti, Y., Liu, S. X., Marancik, D. P., and Wiens, G. D. (2014b). Validation of linked QTL for bacterial cold water disease resistance and spleen size on rainbow trout chromosome Omy19. Aquaculture 432, 139-143. doi: 10.1016/j.aquaculture.2014.05.003

Wiens, G. D., Lapatra, S. E., Welch, T. J., Evenhuis, J. P., Rexroad, C. E., and Leeds, T. D. (2013a). On-farm performance of rainbow trout (Oncorhynchus mykiss) selectively bred for resistance to bacterial cold water disease: effect of rearing environment on survival phenotype. Aquaculture 388, 128-136. doi: 10.1016/j.aquaculture.2013.01.018

Wiens, G. D., Vallejo, R. L., Leeds, T. D., Palti, Y., Hadidi, S., Liu, S., et al. (2013b). Assessment of genetic correlation between bacterial cold water disease resistance and spleen index in a domesticated population of rainbow trout: identification of QTL on chromosome Omy19. PLoS ONE 8:e75749. doi: 10.1371/journal.pone.0075749
Wiens, G. D., Marancik, D. P., Zwollo, P., and Kaattari, S. L. (2015). Reduction of rainbow trout spleen size by splenectomy does not alter resistance against bacterial cold water disease. Dev. Comp. Immunol. 49, 31-37. doi: 10.1016/j.dci.2014.11.003

Conflict of Interest Statement: The authors declare that the research was conducted in the absence of any commercial or financial relationships that could be construed as a potential conflict of interest.

Copyright (c) 2015 Liu, Vallejo, Palti, Gao, Marancik, Hernandez and Wiens. This is an open-access article distributed under the terms of the Creative Commons Attribution License (CC BY). The use, distribution or reproduction in other forums is permitted, provided the original author(s) or licensor are credited and that the original publication in this journal is cited, in accordance with accepted academic practice. No use, distribution or reproduction is permitted which does not comply with these terms. 